\title{
Terapia di prima linea dell'osteoporosi indotta da glucocorticoidi
}

\author{
Nazarena Betella ${ }^{1}$ Miriam Cellini ${ }^{2}$
}

Accettato: 8 agosto 2021 / Pubblicato online: 23 febbraio 2022

(c) The Author(s) 2022

Il quiz n. 135, http://societaitalianadiendocrinologia.it/public/ /pdf/quiz135.pdf, dal titolo "Dottore quanto devo preoccuparmene?" si riferiva a una signora di 54 anni che aveva assunto prednisone $50 \mathrm{mg}$ a dosi scalari per oltre un anno perché affetta da sclerodermia. Con familiarità autoimmune (artrite reumatoide nella nonna) e per frattura a bassa energia (madre), la signora aveva sofferto 15 anni prima di morbo di Basedow trattato e non recidivato. In menopausa da circa tre anni, non ha assunto TOS, non consuma latte e latticini, svolge regolare e intensa attività fisica, non fuma, non beve alcoolici ed è normopeso. Si presenta in ambulatorio con normali valori degli analiti relativi al metabolismo calcio-fosforo, PTH e con carenza di Vit. D e con i seguenti dati di densitometria ossea: T-score rachide lombare $-2,4$ DS, T-score collo femorale $-1,8 \mathrm{DS}$, Tscore femore totale $-1,9$ DS. Oltre allo steroide, la signora sta assumendo un procinetico e un inibitore di pompa protonica, prescritti dal Collega Gastroenterologo dopo evidenza alla manometria di un'alterata peristalsi esofagea e di un'incontinenza dello sfintere esofageo inferiore condizionante un'esofagite da reflusso.

Alla domanda: "Quale, tra i seguenti, rappresenta l'approccio terapeutico più idoneo nella gestione clinica di questa paziente?" soltanto una sparuta minoranza dei numerosi solutori (18\%) ha fornito la risposta n. 3 indicata come corretta: "Terapia di I linea con bisfosfonato ev, da associare a adeguata supplementazione con calcio e vitamina D". La maggioranza dei solutori (53\%) indicava la risposta n. 2: "Terapia di I linea con bisfosfonato orale, da associare a

Proposto da C. Regalbuto.

$凶$ N. Betella

nazarena.betella@humanitas.it

M. Cellini

miriamcellini@yahoo.it

1 U.O. Endocrinologia, Diabetologia e Andrologia Medica, IRCCS Humanitas Research Hospital, Rozzano, MI, Italia

2 S.O.C. di Endocrinologia, Malattie del Metabolismo e Nutrizione Clinica, Azienda Ospedaliero-Universitaria S. Maria della Misericordia, Udine, Italia adeguata supplementazione con calcio e vitamina D", circa un quarto di essi (24\%) la risposta n. 1: "Nessun farmaco osteoattivo, solo supplementazione di calcio e vitamina D; la paziente non presenta infatti fratture e la MOC mostra un quadro di osteopenia iniziale, da rivalutare a 18-24 mesi" e pochissimi $(5 \%)$ la risposta n. 4 : "Terapia anabolica con teriparatide, da associare a adeguata supplementazione con calcio e vitamina D".

L'osteoporosi indotta da glucocorticoidi (GIO), farmaci assunti cronicamente dallo $0,5-2 \%$ della popolazione, è la forma più comune di osteoporosi secondaria. La GIO è caratterizzata da una rapida perdita di massa ossea che, unitamente ad alterazioni della microstruttura scheletrica, coinvolge prevalentemente l'osso trabecolare e insorge precocemente entro i 6-12 mesi dall'avvio della terapia steroidea [1-3]. I meccanismi fisiopatologici della GIO differiscono da quelli dell'osteoporosi primaria post-menopausale e la perdita di massa ossea si verifica attraverso plurimi effetti diretti e indiretti dei glucocorticoidi (GC) sull'osso [1-3]. Nelle fasi precoci di esposizione ai GC si assiste a un incremento dell'attività degli osteoclasti e del riassorbimento osseo, a cui subentra successivamente un'inibizione dell'attività degli osteoblasti con conseguente riduzione di neoapposizione di matrice ossea [1-4]. A questi effetti diretti sul metabolismo scheletrico si aggiungono effetti indiretti e sistemici dei glucocorticoidi (es. inibizione gli ormoni sessuali e dell'asse somatotropo, riduzione dell'assorbimento intestinale e incremento dell'escrezione renale del calcio, riduzione il trofismo muscolare con aumento del rischio di cadute) che concorrono a incrementare ulteriormente il rischio di fratture da fragilità scheletrica [1-4].

Le fratture da fragilità scheletrica in corso di terapia cronica con GC, evento comune temibile, occorrono principalmente a livello vertebrale già a partire dai primi 3-6 mesi dall'avvio della terapia con un'incidenza annua, rispettivamente, del 2,5 e $5,1 \%$ in pazienti neo-trattati ( $\leq 6$ mesi) e in quelli in terapia cronica ( $>6$ mesi) [5]. Tale rischio fratturativo dipende sia dalla dose giornaliera di GC (dosi equivalenti a prednisolone $\geq 7,5 \mathrm{mg} /$ die correlano con un rischio 5 volte maggiore di fratture vertebrali e 2 volte maggiore di 
fratture di femore) che dalla durata complessiva del trattamento [6]. In assenza di un trattamento di prevenzione primaria, oltre il $10 \%$ dei pazienti in terapia cronica con GC svilupperà una frattura clinica da fragilità scheletrica e il $30-40 \%$ dei casi presenterà almeno una frattura radiologica vertebrale asintomatica, soprattutto se coesistono altri fattori di rischio per fragilità scheletrica [7].

Gli eventi fratturativi in corso di terapia con GC, oltre che precocemente, possono verificarsi con valori di BMD normali o solo minimamente ridotti [8] e la valutazione della sola BMD non permette, come per altre forme di osteoporosi secondaria, di identificare tutti i pazienti ad elevato rischio fratturativo [9]. È stato, pertanto, proposto da alcune società scientifiche un valore di $\mathrm{T}$-score più basso $(\mathrm{da}-1 \mathrm{a}$ $-1,5$ DS) come soglia per il trattamento in prevenzione primaria della GIO [3, 10] mentre per le linee guida italiane [8] la terapia cronica con GC per dosi equivalenti a prednisone $\geq 5 \mathrm{mg} / \mathrm{die}$ si associa a un rischio di frattura così elevato che la decisione di avviare un trattamento può prescindere dall' acquisizione dei valori densitometrici.

La valutazione del rischio fratturativo all'avvio del trattamento con GC deve essere eseguita precocemente e la terapia di prevenzione primaria delle fratture in soggetti considerati ad alto rischio deve essere avviata tempestivamente, considerando la rapidità con cui il danno osseo si verifica $[1-3,8,10]$. È ormai accertato che dosi giornaliere equivalenti a prednisolone $\geq 5 \mathrm{mg}$ per almeno 3 mesi correlino con un aumentato rischio fratturativo [3,9]; pertanto, le linee guida nazionali utilizzano questo limite come valore soglia di trattamento [8], mentre altre raccomandano il trattamento con dosaggi maggiori [10]. Inoltre, le principali linee guida raccomandano, nei pazienti in terapia cronica con GC, un appropriato introito di calcio $(\geq 1200 \mathrm{mg} / \mathrm{die})$ e colecalciferolo o equivalenti ( $\geq 400 \mathrm{UI} / \mathrm{die}$ ), un adeguato apporto energetico-proteico e modifiche dello stile di vita con adeguato esercizio fisico (per contrastare la perdita della massa muscolare) e astensione da fumo e alcol [1-3, 8, 10-12].

Sebbene l'integrazione di calcio e vitamina D abbia dimostrato efficacia nel prevenire la perdita di mineralizzazione ossea a livello lombare e dell'avambraccio, nessun beneficio è stato riportato sulla BMD femorale, nella riduzione dei markers di turn-over osseo e del rischio di frattura [13]. Al contrario, la terapia con bisfosfonati (BP), associata a supplementazione di calcio e vitamina $\mathrm{D}$, si è dimostrata superiore alla sola terapia con calcio e vitamina D nel migliorare la BMD e nel ridurre il rischio fratturativo [14]. In accordo con le raccomandazioni delle principali Società Scientifiche Nazionali e Internazionali (Società Italiana dell'Osteoporosi, del Metabolismo Minerale e delle Malattie dello Scheletro, SIOMMMS; American College of Rheumatology, ACR; National Osteoporosis Guideline Group, NOGG; International Osteoporosis Foundation, IOF) [8, 10-12], l'impiego di bisfosfonati orali o endovena rappresenta il trattamento di I linea in caso di esposizione a dosi di prednisone $>5-7,5 \mathrm{mg} /$ die o equivalenti per una durata $>3$ mesi in assenza di fratture da fragilità preesistenti, considerando il basso costo e il profilo di sicurezza-efficacia di questi farmaci.

Nella GIO, tutti i bisfosfonati hanno dimostrato efficacia sulla BMD lombare e femorale sia in prevenzione primaria che secondaria $[15,16]$ con evidenza di una riduzione dell'incidenza di fratture vertebrali per alendronato e risedronato [17]. In studi comparativi, acido zoledronico [18] e teriparatide [19] si sono dimostrati superiori sull'outcome primario della preservazione di massa ossea rispetto a risedronato e alendronato, rispettivamente. Nel primo caso [18], il tasso di frattura era troppo basso per valutarne l'efficacia anti-fratturativa, mentre nel secondo caso [19], sebbene l'evento fratturativo non fosse un end-point primario dello studio, si è verificato un numero significativamente inferiore di fratture vertebrali incidenti nei pazienti trattati con teriparatide rispetto a quelli trattati con alendronato. Mentre i bisfosfonati, molto probabilmente in ragione della loro azione anti-riassorbitiva, sono generalmente più efficaci nelle donne in post-menopausa rispetto alle donne in pre-menopausa in termini di incremento della BMD, teriparatide ha dimostrato un aumento sovrapponibile della BMD lombare nelle donne in pre- e post-menopausa [19].

Questi dati, unitamente al meccanismo fisiopatologico di ridotta neoformazione ossea predominante nella GIO, hanno indotto alcuni autori a suggerire di considerare la terapia anabolica con teriparatide un'opzione terapeutica di prima linea nei pazienti a rischio particolarmente elevato (es. pazienti anziani che iniziano terapia GC ad elevato dosaggio, presenza di pregresse fratture multiple e densità minerale ossea iniziale molto ridotta). Secondo le raccomandazioni delle linee guida italiane [8] e i criteri di rimborsabilità per il trattamento della GIO [20], donne in post-menopausa e uomini $>50$ anni senza storia di frattura da fragilità, in cui è atteso un trattamento di $>3$ mesi con prednisone $\geq 5 \mathrm{mg} / \mathrm{die}$ o equivalenti devono essere trattati con bisfosfonati orali o endovena (se precedenti controindicati o non tollerati) come prima linea e con denosumab come seconda linea di trattamento. La terapia anabolica con teriparatide, seppur allettante sia per la patogenesi della GIO sia per la superiorità nella prevenzione delle fratture da fragilità scheletrica in confronto ai bisfosfonati in questo contesto clinico [1-3, 19], rientra nei criteri di rimborsabilità secondo nota AIFA 79 solo in presenza di frattura vertebrale o femorale in corso di terapia con $\mathrm{GC} \geq 12$ mesi alla dose di prednisone o equivalenti $\geq 5 \mathrm{mg} /$ die [20].

\section{Dichiarazioni etiche}

Conflitto di interesse Le autrici Nazarena Betella e Miriam Cellini dichiarano di non avere conflitti di interesse. 
Consenso informato Lo studio presentato in questo articolo non ha richiesto sperimentazione umana.

Studi sugli animali Le autrici di questo articolo non hanno eseguito studi sugli animali.

Open Access This article is licensed under a Creative Commons Attribution 4.0 International License, which permits use, sharing, adaptation, distribution and reproduction in any medium or format, as long as you give appropriate credit to the original author(s) and the source, provide a link to the Creative Commons licence, and indicate if changes were made. The images or other third party material in this article are included in the article's Creative Commons licence, unless indicated otherwise in a credit line to the material. If material is not included in the article's Creative Commons licence and your intended use is not permitted by statutory regulation or exceeds the permitted use, you will need to obtain permission directly from the copyright holder. To view a copy of this licence, visit http://creativecommons.org/licenses/by/4.0/.

\section{Bibliografia}

1. Adami G, Saag KG (2019) Glucocorticoid-induced osteoporosis: 2019 concise clinical review. Osteoporos Int 30(6):1145-1156

2. Compston J (2018) Glucocorticoid-induced osteoporosis: an update. Endocrine 61(1):7-16

3. Kenanidis E, Potoupnis ME, Kakoulidis P et al (2015) Management of glucocorticoid-induced osteoporosis: clinical data in relation to disease demographics, bone mineral density and fracture risk. Expert Opin Drug Saf 14(7):1035-1053

4. Canalis E, Mazziotti G, Giustina A, Bilezikian JP (2007) Glucocorticoid-induced osteoporosis: pathophysiology and therapy. Osteoporos Int 18(10):1319-1328

5. Amiche MA, Albaum JM, Tadrous M et al (2016) Fracture risk in oral glucocorticoid users: a Bayesian meta-regression leveraging control arms of osteoporosis clinical trials. Osteoporos Int 27(5):1709-1718

6. Van Staa TP, Leufkens HG, Abenhaim L et al (2000) Use of oral corticosteroids and risk of fractures. $\mathrm{J}$ Bone Miner Res 15(6):993-1000

7. Angeli A, Guglielmi G, Dovio A et al (2006) High prevalence of asymptomatic vertebral fractures in post-menopausal women receiving chronic glucocorticoid therapy: a cross-sectional outpatient study. Bone 39(2):253-259

8. Rossini M, Adami S, Bertoldo F et al (2016) Guidelines for the diagnosis, prevention and management of osteoporosis. Reumatismo 68:1-39
9. van Staa TP, Leufkens HG, Cooper C (2002) The epidemiology of corticosteroid-induced osteoporosis: a meta-analysis. Osteoporos Int 13(10):777-787

10. Buckley L, Guyatt G, Fink HA et al (2017) 2017 American College of Rheumatology guideline for the prevention and treatment of glucocorticoid-induced osteoporosis. Arthritis Care Res (Hoboken) 69(8):1095-1110

11. Compston J, Cooper A, Cooper C et al (the National Osteoporosis Guideline Group (NOGG)) (2017) UK clinical guideline for the prevention and treatment of osteoporosis. Arch Osteoporos 12(1):43

12. Lekamwasam $\mathrm{S}$, Adachi JD, Agnusdei $\mathrm{D}$ et al(the Joint IOF-ECTS GIO Guidelines Working Group) (2012) A framework for the development of guidelines for the management of glucocorticoid-induced osteoporosis. Osteoporos Int 23(9):2257-2276

13. Homik J, Suarez-Almazor ME, Shea B et al (2000) Calcium and vitamin D for corticosteroid-induced osteoporosis. Cochrane Database Syst Rev 1998(2):CD000952

14. Amin S, LaValley MP, Simms RW, Felson DT (1999) The role of vitamin D in corticosteroid-induced osteoporosis: a meta-analytic approach. Arthritis Rheum 42(8): 1740-1751

15. Stoch SA, Saag KG, Greenwald M et al (2009) Once-weekly oral alendronate $70 \mathrm{mg}$ in patients with glucocorticoid-induced bone loss: a 12-month randomized, placebo-controlled clinical trial. J Rheumatol 36(8):1705-1714

16. Cohen S, Levy RM, Keller M et al (1999) Risedronate therapy prevents corticosteroid-induced bone loss: a twelve-month. Multicenter, randomized, double-blind, placebo-controlled, parallel-group study. Arthritis Rheum 42(11):2309-2318

17. Wallach S, Cohen S, Reid DM et al (2000) Effects of risedronate treatment on bone density and vertebral fracture in patients on corticosteroid therapy. Calcif Tissue Int 67(4):277-285

18. Reid DM, Devogelaer JP, Saag K (the HORIZON investigators) (2009) Lancet 373(9671):1253-1263

19. Saag KG, Zanchetta JR, Devogelaer JP et al (2009) Effects of teriparatide versus alendronate for treating glucocorticoidinduced osteoporosis: thirty-six-month results of a randomized, double-blind, controlled trial. Arthritis Rheum 60(11):3346-3355

20. https://www.aifa.gov.it/nota-79. Accessed on 13-11-2021

Nota della casa editrice Springer Nature rimane neutrale in riguardo alle rivendicazioni giurisdizionali nelle mappe pubblicate e nelle affiliazioni istituzionali. 